\title{
Performance enhancement of cooperative networks utilizing MAP decoder and Alamouti code
}

\author{
Safwat M. Ramzy \\ dept. Electrical Engineeing, Faculty of \\ Engineering Sohag University \\ Sohag, Egypt \\ safwat.ramzy@eng.sohag.edu.eg
}

\begin{abstract}
In this paper, we introduced another methodology for the cooperative network using the maximum posterior (MAP) and the Alamouti code decoding scheme for the multiple-input single-output (MISO) wireless networks that use decode and forward (DF) protocol as a cooperation protocol. Without loss of generality, the considered network consists of one source, one relay and one receiver. A closedform expression for the upper bound of the bit error probability is derived. The obtained upper bound expression can be utilized in the power optimization problems, relay positioning issue. The results that are shown in this paper clear that the proposed scheme has two advantages over the related work. The first advantage is that it has less complexity. The second one is that it has better spectrum efficiency by using less number of channels. Therefore, our contribution can be summarized in improving the spectrum efficiency and reducing the complexity of the cooperative network, but the paid price is that the bit error rate increases by a little ratio.
\end{abstract}

Keywords-MAP decoder, Cooperative network, Alamouti code.

\section{Introduction}

Cooperative communication has gained a significant popularity in the last years where the network devices cooperate with each other to improve the performance of the wireless network [1:3]. Cooperative relaying makes use of the broadcasting process related to the wireless system where the transmitted signals can be received, modified, and forwarded by any device in the source coverage area to the desired receiver. In the cooperative diversity, relay devices forward the provided signal from the source to the desired receiver, enabling the receiver to overcome channel impairments caused by fading and other noise [1]. The literature has suggested several communication protocols [4], such as decode and forward (DF), amplify and forward (AF), and compress and forward (CF). Regarding the work under consideration, we consider the decoding and forwarding (DF) protocol. In DF protocol, the transfer of data takes place over two stages. In the first stage, the source of data sends its data to the desired receiver. The data sent by the source naturally, inthe wireless medium, may be received via relay devices. $R$ elay devices decode the received symbols in the second stage and then the relays retransmit the decoded signals to the desired receiver. Because the link between the source and the relay is not always completely perfect, there may be an introduced error from the relay side. Therefore, the signals that reach the receiver from the relay may have some errors regarding the source data. There are many approaches have been presented to allow the receiver to recover the data sent by the source [5-6].

These combination strategies could provide a link quality similar to that given by conventional diversity schemes if the source-relay path (S-R channel) is error-free; otherwise, it will proceed to error propagation problem [7]. In the literature, various approaches [8-9] were introduced to reconstruct the data at the receiver by taking the error in the S-R channel into account. The authors in [10] developed an analytical formula for the symbol error rate of the DF protocol in Rayleigh's fading system for M-ary phase-shift keying system. The basic concept of network coding [11-12] is to code the data at the intermediate device. Recently, network coding on GF (2) (i.e., XOR-only coding) is used to improve end-to-end performance in multi-hop wireless networks when it is implemented above the IEEE 802.11 MAC layer [13].

In this work, an integration between [14] and [15] with making use of the advantages of each one is done. Therefore, we propose a new approach using a decoding scheme that utilizes both Alamouti combining (AC) code and maximum posterior (MAP) decoder to recover the data at the receiver. In [15], after the relay decodes the two received symbols from the source; it sends the data to the receiver one symbol per time slot. Therefore, two time slots are needed to send the data from the relay in [15] to the desired receiver. Our proposed scheme uses the cooperative network coding. After the relay decodes the two symbols sent by the source using Alamouti combining, it encodes (linearly combine) the decoded two symbols to generate the parity bit that is forwarded to the receiver in a time slot. The MAP decoder estimates the data sent by the source according to the three signals that come from both the source and the relay as shown in the next sections. A closed formula for the upper bound on the bit error rate of the proposed scheme is introduced. The derived upper bound considers all variations of the channels (i.e. source-to-relay, source-to-receiver, and relay-to-receiver). The obtained upper bound could be used to enhance the system performance with the effective use of available power capabilities.

The rest of this paper is organized as follows. The system model is introduced in section II. The proposed approach is given in section III. The bit error rate of the proposed approach is introduced in section IV. The 
simulation results are drawn in section V. The conclusions are outlined in section VI.

\section{System model}

We consider the same relay network found in [15] that consists of a source (S) having two antennas, a relay (R), and a receiver (D) that is described in Figure 1. The transmission process occurs in two stages. In the first stage, the source transmits its data to the receiver utilizing the Alamouti code [15] to obtain the desired diversity. Utilizing the given model, two symbols $\mathrm{s}_{1}$ and $\mathrm{s}_{2}$ are sent simultaneously twice in two time slots $t_{1}$ and $t_{2}$. In time slot $t_{1}$, the signals corresponding to $s_{1}$ and $s_{2}$ are sent from the two antennas of the source.

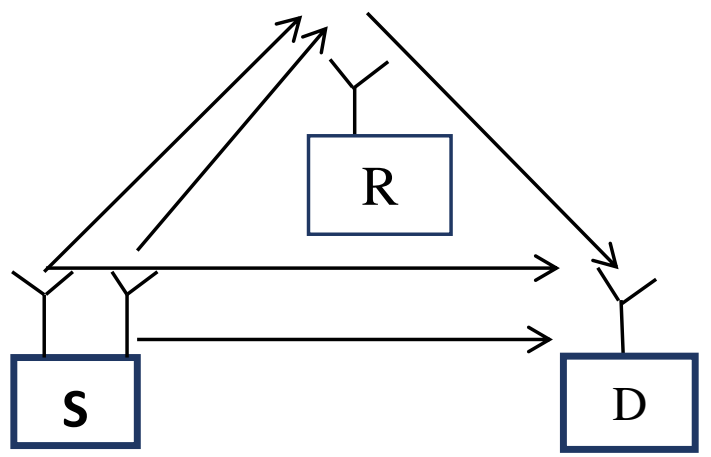

Fig. 1 System model

In the time slot $t_{2}$, the two antennas send signals corresponding to $-s_{2}^{*}$ and $s_{1}^{*}$, respectively, where "*" denotes the complex conjugate. These data that are sent by the source are received by the relay with some errors according to the channel quality between the source and the relay. Assuming that BPSK modulation is used here and the source generates its bits with the same probability, i.e. $p\left(b_{i}=0\right)=p\left(b_{i}=1\right)=.5$ where $b_{i}$ is the source bit for $i=1,2$. It is assumed that all channels are flat Rayleigh with white Gaussian noise. The channel fading gains are assumed to be unchanged over two successive time slots because of the requirement of the decoding process of Alamouti code [16]. The signals received at the end of the first stage [15] (two time slots) by the relay and the receiver, respectively, are given by

$$
\begin{aligned}
& q_{s r 1}=g_{s r 1} \sqrt{d_{s r}^{-m}} s_{1}+g_{s r 2} \sqrt{d_{s r}^{-m}} s_{2}+n_{r 1} \\
& q_{s r 2}=-g_{s r 1} \sqrt{d_{s r}^{-m}} s_{2}^{*}+g_{s r 2} \sqrt{d_{s r}^{-m}} s_{1}^{*}+n_{r 2} \\
& q_{s d 1}=g_{s d 1} \sqrt{d_{s d}^{-m}} s_{1}+g_{s d 2} \sqrt{d_{s d}^{-m}} s_{2}+n_{s d 1} \\
& q_{s d 2}=-g_{s d 1} \sqrt{d_{s d}^{-m}} s_{2}^{*}+g_{s d} \sqrt{d_{s d}^{-m}} s_{1}^{*}+n_{s d 2}
\end{aligned}
$$

where

- $\mathrm{q}_{\mathrm{sr} 1}$ is the received signal by the relay in the first stage in time slot $t_{1}$

- $\mathrm{q}_{\mathrm{sr} 2}$ is the received signal by the relay in the first stage in time slot $t_{2}$.

- $\mathrm{q}_{\mathrm{sd} 1}$ is the received signal by the receiver in the first stage in time slot $t_{1}$.

- $\mathrm{q}_{\mathrm{sd} 2}$ is the received signal by the receiver in the first stage in time slot $\mathrm{t}_{2}$

- $\mathrm{s}_{\mathrm{i}} \in\left\{+\sqrt{\mathrm{E}_{\mathrm{s}}},-\sqrt{\mathrm{E}_{\mathrm{s}}}\right\}$ is the $i-t h$ modulated symbol sent by the source, $i \in\{1,2\}$ where $E_{s}$ is the energy per symbol of the source.
- $\mathrm{g}_{\text {sri }} \operatorname{andg}_{\text {sdi }} \mathrm{i} \in\{1,2\}$ are the fading gains of the S-R and $\mathrm{S}-\mathrm{D}$ channels, respectively.

- $\mathrm{d}_{\mathrm{sr}}$ is the distance between the source and the relay.

- $\mathrm{d}_{\mathrm{sd}}$ is the distance between the source and the receiver.

- $\mathrm{m}$ is the path loss exponent.

- $\mathrm{n}_{\mathrm{ri}} \mathrm{i} \in\{1,2\}$ is the white Gaussian noise at the relay with zero mean and variance $\mathrm{N}_{0} / 2$.

- $\mathrm{n}_{\text {sdi }} \mathrm{i} \in\{1,2\}$ is the white Gaussian noise at the receiver with zero mean and variance $\mathrm{N}_{0} / 2$.

In the second stage, when the relay receives the signals from the source using Alamouti code, the relay estimates the two symbols sent by the source by calculating the quantities $\mathrm{q}_{1}$ and $\mathrm{q}_{1}$ according to:

$\mathrm{q}_{1}=\mathrm{g}_{\mathrm{sr} 1}^{*} \mathrm{q}_{\mathrm{sr} 1}+\mathrm{g}_{\mathrm{sr} 2} \mathrm{q}_{\mathrm{sr} 2}^{*}$

$\mathrm{q}_{2}=\mathrm{g}_{\mathrm{sr} 2}^{*} \mathrm{q}_{\mathrm{sr} 1}-\mathrm{g}_{\mathrm{sr} 1} \mathrm{q}_{\mathrm{sr} 2}^{*}$

Then the relay estimates the decoded bits as follows

$\mathrm{x}_{\mathrm{ri}}= \begin{cases}0 & \mathrm{q}_{\mathrm{i}} \geq 0 \\ 1 & \mathrm{q}_{\mathrm{i}}<0\end{cases}$

where $x_{r i}$ is the estimation of the source bit $b_{i}$ that is received by the relay then the relay encodes the decoded bits to generate a parity bit. Let $\mathrm{P}_{\mathrm{r}} \in\{0,1\}$ is the bit sent by the relay. In GF (2), 0 is the additive identity element under $\bigoplus$ (modulo- 2 ) addition. The parity bit sent by the relay is given by

$\mathrm{P}_{\mathrm{r}}=\mathrm{x}_{\mathrm{r} 1} \oplus \mathrm{x}_{\mathrm{r} 2}$

The signals received at the receiver sent by the relay are given by

$\mathrm{q}_{\mathrm{rd}}=\mathrm{g}_{\mathrm{rd}} \sqrt{\mathrm{d}_{\mathrm{rd}}^{-\mathrm{m}}} \mathrm{s}_{\mathrm{r}}+\mathrm{n}_{\mathrm{rd}}$

where

- $\mathrm{q}_{\mathrm{rd}}$ is the signal received by the receiver that is sent by the relay in the second stage.

- $\mathrm{s}_{\mathrm{r}} \in\left\{+\sqrt{\mathrm{E}_{\mathrm{r}}},-\sqrt{\mathrm{E}_{\mathrm{r}}}\right\}$ is the $i-t h$ modulated symbol sent by the relay corresponding to $\mathrm{P}_{\mathrm{r}}$ where $\mathrm{E}_{\mathrm{r}}$ is the energy per symbol of the relay.

- $\mathrm{g}_{\mathrm{rd}}$ is the fading gain of the R-D link.

- $\mathrm{d}_{\mathrm{rd}}$ is the distance between the relay and the receiver.

- $\mathrm{n}_{\mathrm{rd}}$ is the white Gaussian noise of at the receiver with zero mean and variance $\mathrm{N}_{0} / 2$.

Let $\mathrm{e}_{\mathrm{ri}} \in\{0,1\}$ be the error of the received source bit at the relay for $\mathrm{i} \epsilon\{1,2\}$, i.e. $\mathrm{x}_{\mathrm{ri}}=\mathrm{b}_{\mathrm{i}} \oplus \mathrm{e}_{\mathrm{ri}}$, where $\mathrm{e}_{\mathrm{ri}}=1$ means $x_{r i} \neq b_{i}$, i.e. $b_{i}$ is decoded in error at the relay, and $e_{r i}=0$ means $\mathrm{x}_{\mathrm{ri}}=\mathrm{b}_{\mathrm{i}}$. Then, (8) can be written as

$\mathrm{P}_{\mathrm{r}}=\mathrm{b}_{1} \oplus \mathrm{b}_{2} \oplus \mathrm{e}_{\mathrm{r} 1} \oplus \mathrm{e}_{\mathrm{r} 2}$

$\mathrm{P}_{\mathrm{r}}=\mathrm{b}_{1} \oplus \mathrm{b}_{2} \oplus \mathrm{zr}$

where

$\mathrm{zr}=\mathrm{e}_{\mathrm{r} 1} \bigoplus \mathrm{e}_{\mathrm{r} 2}$

Therefore, zr represents the expected error that occurs in the source-to-relay link. Let $\mathrm{p}_{\mathrm{ir}}$ be the probability of the error of the $i-t h$ source bit received by the relay. Then, the event $\mathrm{zr}=1$, i.e. wrong decoding (forwarding error), occurs if only one of $e_{\mathrm{r} 2}$ or $\mathrm{e}_{\mathrm{r} 2}$ is 1 . Hence, the probability of $\mathrm{zr}$ can be written as

$\mathrm{p}(\mathrm{zr}=1)=\mathrm{p}_{1 \mathrm{r}}\left(1-\mathrm{p}_{2 \mathrm{r}}\right)+\mathrm{p}_{2 \mathrm{r}}\left(1-\mathrm{p}_{1 \mathrm{r}}\right)$ 
$\mathrm{p}(\mathrm{zr}=0)=1-\mathrm{p}(\mathrm{zr}=1)$

Where $\mathrm{p}_{\text {ir }}$ is given by [16]

$\mathrm{p}_{\mathrm{ir}}=.25-\sqrt{\frac{\gamma_{\mathrm{sr}}}{1.5+\gamma_{\mathrm{sr}}}}+.75 \sqrt{\frac{\gamma_{\mathrm{sr}}}{2+\gamma_{\mathrm{sr}}}}(15)$

where $\gamma_{s r}=d_{s r}^{-m} E_{S} / N_{o}$ is the average received SNR of the source - relay link.

Table 1: Transmitter Side code book

\begin{tabular}{|c|c|c|c|}
\hline & $\mathbf{z r}=\mathbf{0}$ & & $\mathbf{z r}=\mathbf{1}$ \\
\hline $\mathbf{w}_{\mathbf{0}}$ & 000 & $\mathbf{w}_{\mathbf{1}}$ & 001 \\
\hline $\mathbf{w}_{\mathbf{3}}$ & 011 & $\mathbf{w}_{\mathbf{2}}$ & 010 \\
\hline $\mathbf{w}_{\mathbf{5}}$ & 101 & $\mathbf{w}_{\mathbf{4}}$ & 100 \\
\hline $\mathbf{w}_{\mathbf{6}}$ & 110 & $\mathbf{w}_{\mathbf{7}}$ & 111 \\
\hline
\end{tabular}

Table 1 shows the transmitter code book for all possible values of zr. The first and the second bits of the codeword represent the source data while the third bit of thecodeword is the bit that is generated by the relay. For example, if the transmitted codeword is 101 then $\mathrm{b}_{1}=1, \mathrm{~b}_{2}=0$, and $\mathrm{P}_{\mathrm{r}}=$ 1.The signals received by the receiver can be written in the matrix form as follows [7]

$\mathrm{Q}=\mathrm{GT}+\mathrm{n}$

Where $Q=\left[\begin{array}{lll}\mathrm{q}_{\mathrm{sd} 1} & \mathrm{q}_{\mathrm{sd} 2}^{*} & \mathrm{q}_{\mathrm{rd}}\end{array}\right]^{\mathrm{T}}, \quad \mathrm{T}=\left[\begin{array}{lll}\mathrm{s}_{1} & \mathrm{~s}_{2} & \mathrm{~s}_{\mathrm{r}}\end{array}\right]^{\mathrm{T}}$ $\mathrm{n}=\left[\begin{array}{lll}\mathrm{n}_{\mathrm{sd} 1} & \mathrm{n}_{\mathrm{sd} 2}^{*} & \mathrm{n}_{\mathrm{rd}}\end{array}\right]$, and Gis given by:

$G=\left[\begin{array}{llr}g_{s d 1} \sqrt{d_{s d}^{-m}} & g_{s d} \sqrt{d_{s d}^{-m}} & 0 \\ g_{s d 2}^{*} \sqrt{d_{s d}^{-m}} & -g_{s d}^{*} \sqrt{d_{s d}^{-m}} & 0 \\ 0 & 0 & g_{r d} \sqrt{d_{r d}^{-m}}\end{array}\right]$

\section{The proposed decoding scheme}

In the considered network, we present the MAP decoder for the MISO network. The MAP decoder is optimal since it minimizes the error rate. The transmitted codeword is estimated by:

$$
\widehat{\mathrm{w}}=\arg \max _{\mathrm{w}_{\mathrm{i}}} \mathrm{p}\left(\mathrm{w}_{\mathrm{i}} \mid \mathrm{Q}\right)
$$

Equation (20) can be written as follows

$\widehat{\mathrm{w}}=\arg \max _{\mathrm{w}_{\mathrm{i}}} \frac{\mathrm{p}\left(\mathrm{Q} \mid \mathrm{w}_{\mathrm{i}}\right) \mathrm{P}\left(\mathrm{w}_{\mathrm{i}}\right)}{\mathrm{P}(\mathrm{Q})}=\arg \max _{\mathrm{w}_{\mathrm{i}}} \mathrm{p}\left(\mathrm{Q} \mid \mathrm{w}_{\mathrm{i}}\right) \mathrm{p}\left(\mathrm{w}_{\mathrm{i}}\right)(19)$ $\left(\mathrm{Q} \mid \mathrm{w}_{\mathrm{i}}\right)=\frac{1}{\left(\pi \mathrm{N}_{0}\right)^{3 / 2}} \mathrm{e}^{-\left\|\mathrm{Q}-\mathrm{GT}_{\mathrm{i}}\right\|^{2}} / \mathrm{N}_{0}(20)$

Equation (20) comes from the joint distribution of Gaussian random variables [1] and $T_{i}$ is the modulated vector corresponding to codeword $\mathrm{w}_{\mathrm{i}}$ for $\mathrm{w}_{1}=\left[\begin{array}{lll}0 & 0 & 1\end{array}\right], \mathrm{T}_{1}=$ $\left[+\sqrt{E_{1}}+\sqrt{E_{2}}-\sqrt{E_{r}}\right]$. Since $p(z r)$ is related to the amount of error of the link between the source and the relay. The MAP decoder should initially calculate the probability $\mathrm{p}\left(\mathrm{w}_{\mathrm{i}}\right)$ of all the codewords and then it estimates the transmitted codeword. Substituting from (20) into (19) yields

$$
\begin{aligned}
\widehat{\mathrm{w}} & =\arg \max _{\mathrm{w}_{\mathrm{i}}} \mathrm{p}\left(\mathrm{w}_{\mathrm{i}}\right) \frac{1}{\left(\pi \mathrm{N}_{0}\right)^{3 / 2}} \mathrm{e}^{-\left\|\mathrm{Q}-\mathrm{GT}_{\mathrm{i}}\right\|^{2}} / \mathrm{N}_{0} \\
& =\arg \min _{\mathrm{w}_{\mathrm{i}}}\left\|\mathrm{Q}-\mathrm{GT}_{\mathrm{i}}\right\|^{2}-\mathrm{N}_{0} \log \left(\mathrm{P}\left(\mathrm{w}_{\mathrm{i}}\right)\right)
\end{aligned}
$$

The probability $\mathrm{p}\left(\mathrm{w}_{\mathrm{i}}\right)$ is given by

$$
\mathrm{p}\left(\mathrm{w}_{0}\right)=\mathrm{p}\left(\mathrm{b}_{1}=0\right) \times \mathrm{p}\left(\mathrm{b}_{2}=0\right) \times \mathrm{p}\left(\mathrm{P}_{\mathrm{r}}=0 \mid \mathrm{b}_{1}=0, \mathrm{~b}_{2}=\right.
$$

According to (11), the event $\mathrm{p}\left(\mathrm{P}_{\mathrm{r}}=0 \mid \mathrm{b}_{1}=0, \mathrm{~b}_{2}=0\right)$ occurs when $\mathrm{zr}=0$ Then, $\mathrm{p}\left(\mathrm{P}_{\mathrm{r}}=0 \mid \mathrm{b}_{1}=0, \mathrm{~b}_{2}=0\right)=$ $\mathrm{p}(\mathrm{zr}=0)$, so $(22)$ can be written as follows

$\mathrm{p}\left(\mathrm{w}_{0}\right)=\mathrm{p}\left(\mathrm{b}_{1}=0\right) \times \mathrm{p}\left(\mathrm{b}_{2}=0\right) \times \mathrm{p}(\mathrm{zr}=0)$

$$
=\frac{1}{2} \times \frac{1}{2} \times \mathrm{p}(\mathrm{zr}=0)=\frac{1}{4} \times \mathrm{p}(\mathrm{zr}=0)
$$

In general, the probability of transmitting the codeword $\mathrm{w}_{\mathrm{i}}$ can be written as

$p\left(w_{i}\right)= \begin{cases}\frac{1}{4} \times p(z r=0), & \text { for } \mathrm{i}=0,3,6,5 \\ \frac{1}{4} \times p(z r=1), & \text { for } \mathrm{i}=1,2,4,7\end{cases}$

Where $\mathrm{p}(\mathrm{zr}=1)$ is given by (13).

\section{Bit error probability calculation of the proposed scheme}

Our target now is to derive the upper bound of the bit error probability of the proposed approach. The upper bound of the bit error probability is given by

$\mathrm{p}_{\mathrm{E}} \leq \frac{1}{2} \sum_{\mathrm{m}=1}^{2} \sum_{\mathrm{k}=0}^{8} \sum_{\substack{\mathrm{j}=0 \\ \mathrm{w}_{j m} \neq w_{k m}}}^{8} \mathrm{p}\left(\mathrm{w}_{\mathrm{k}} \rightarrow \mathrm{w}_{\mathrm{j}}\right) \mathrm{p}\left(\mathrm{w}_{\mathrm{k}}\right)$

where $\mathrm{w}_{\mathrm{jm}}$ is the $m-t h$ bit of the codeword $\mathrm{w}_{\mathrm{j}}$ and $\mathrm{p}\left(\mathrm{w}_{\mathrm{k}} \rightarrow \mathrm{w}_{\mathrm{j}}\right)$ is [14] the probability that the decoding codeword is $\mathrm{w}_{\mathrm{j}}$ when the transmitted codeword is $\mathrm{w}_{k}$ and when these are the only two hypothesis. If the codeword $w_{k}$ is transmitted, the received signals are given by

$$
\mathrm{Q}_{\mathrm{k}}=\mathrm{GT}_{\mathrm{k}}+\mathrm{n}
$$

From (26) and (21), the probability $\mathrm{p}\left(\mathrm{w}_{\mathrm{k}} \rightarrow \mathrm{w}_{\mathrm{j}}\right)$ is given by

$$
\begin{gathered}
\mathrm{p}\left(\mathrm{w}_{\mathrm{k}} \rightarrow \mathrm{w}_{\mathrm{j}}\right)=\mathrm{p}\left(\left(\left\|\mathrm{Q}_{\mathrm{k}}-\mathrm{GT}_{\mathrm{j}}\right\|^{2}\right)-\mathrm{N}_{0} \log \left(\mathrm{p}\left(\mathrm{w}_{\mathrm{j}}\right)\right)\right) \\
\left.<\left(\left\|\mathrm{Q}_{\mathrm{k}}-\mathrm{GT}_{\mathrm{k}}\right\|^{2}\right)-\mathrm{N}_{0} \log \left(\mathrm{p}\left(\mathrm{w}_{\mathrm{k}}\right)\right)\right) \\
=\mathrm{p}\left(\left\|\mathrm{G}\left(\mathrm{T}_{\mathrm{k}}-\mathrm{T}_{\mathrm{j}}\right)+\mathrm{n}\right\|^{2}+\mathrm{N}_{0} \log \frac{\mathrm{p}\left(\mathrm{w}_{\mathrm{k}}\right)}{\mathrm{p}\left(\mathrm{w}_{\mathrm{j}}\right)}<\|\mathrm{n}\|^{2}\right) \\
=\mathrm{p}\left(\left\langle\mathrm{n}, \mathrm{G}\left(\mathrm{w}_{\mathrm{k}}-\mathrm{w}_{\mathrm{j}}\right)\right\rangle>\frac{\left\|\mathrm{G}\left(\mathrm{T}_{\mathrm{k}}-\mathrm{T}_{\mathrm{j}}\right)\right\|^{2}}{2}+\frac{\mathrm{N}_{0}}{2} \log \frac{\mathrm{p}\left(\mathrm{w}_{\mathrm{k}}\right)}{\mathrm{p}\left(\mathrm{w}_{\mathrm{j}}\right)}\right)(27)
\end{gathered}
$$

where $\left\langle n, G\left(w_{k}-w_{j}\right)\right\rangle$ is a Gaussian random variable that has zero mean and variance $\frac{\mathrm{N}_{0}}{2}\left\|\mathrm{G}\left(\mathrm{T}_{\mathrm{k}}-\mathrm{T}_{\mathrm{j}}\right)\right\|^{2}$, accordingly

$$
\mathrm{p}\left(\mathrm{w}_{\mathrm{k}} \rightarrow \mathrm{w}_{\mathrm{j}} \mid \mathrm{h}\right)=\mathrm{Q}\left(\frac{\left\|\mathrm{G}\left(\mathrm{T}_{\mathrm{k}}-\mathrm{T}_{\mathrm{j}}\right)\right\|}{\sqrt{2 \mathrm{~N}_{0}}}+\frac{2 \mu_{\mathrm{kj}} \sqrt{2 \mathrm{~N}_{0}}}{\left\|\mathrm{G}\left(\mathrm{T}_{\mathrm{k}}-\mathrm{T}_{\mathrm{j}}\right)\right\|}\right)
$$

where $\mu_{\mathrm{kj}}=.25 \log \frac{\mathrm{p}\left(\mathrm{w}_{\mathrm{k}}\right)}{\mathrm{p}\left(\mathrm{w}_{\mathrm{j}}\right)}$. The pairwise error probability $\mathrm{p}\left(\mathrm{w}_{\mathrm{k}} \rightarrow \mathrm{w}_{\mathrm{j}}\right)$ is determined by averaging (28) with respect to the joint distribution of fading gains.Let us define $\Psi_{\mathrm{k}}\left(\mu_{\mathrm{ij}}\right)$ as follows

$$
\Psi_{\mathrm{k}}\left(\mu_{\mathrm{ij}}\right)=\mathrm{p}\left(\mathrm{w}_{\mathrm{i}} \rightarrow \mathrm{w}_{\mathrm{j}}\right)\{\forall \mathrm{i}, \mathrm{j} \in\{0,1,2,3,4,5,6,7\}(29)
$$

If $\mathrm{i}=0$ then, 


$$
p\left(w_{0} \rightarrow w_{j}\right)=\left\{\begin{array}{cl}
\Psi_{1}\left(\mu_{0 j}\right) & \text { for } \mathrm{j}=1 \\
\Psi_{2}\left(\mu_{0 j}\right) & \text { for } \mathrm{j}=2,4 \\
\Psi_{3}\left(\mu_{0 \mathrm{j}}\right) & \text { for } \mathrm{j}=3,5 \\
\Psi_{4}\left(\mu_{0 \mathrm{j}}\right) & \text { for } \mathrm{j}=6 \\
\Psi_{5}\left(\mu_{0 \mathrm{j}}\right) & \text { for } \mathrm{j}=7
\end{array}\right.
$$

- Derivation of $\Psi_{1}\left(\mu_{0 j}\right)$ : Substituting $i=0$ and $j=1$ into (28) yields

$\left\|\mathrm{G}\left(\mathrm{T}_{0}-\mathrm{T}_{1}\right)\right\|$

$$
\begin{gathered}
=\left\|\left[\begin{array}{llr}
g_{s d 1} \sqrt{d_{s d}^{-m}} & g_{s d} \sqrt{d_{s d}^{-m}} & 0 \\
g_{s d}^{*} \sqrt{d_{s d}^{-m}} & -g_{s d}^{*} \sqrt{d_{s d}^{-m}} & 0 \\
0 & 0 & g_{r d} \sqrt{d_{r d}^{-m}}
\end{array}\right]\left[\begin{array}{lll}
0 & 0 & 2 \sqrt{E_{r}}
\end{array}\right]^{T}\right\| \\
=\sqrt{2 g_{r d}^{2} d_{r d}^{-m} E_{r}}
\end{gathered}
$$

Accordingly, $\Psi_{1}\left(\mu_{0 \mathrm{j}}\right)$ is given by

$\Psi_{1}\left(\mu_{0 \mathrm{j}}\right)=\mathrm{Q}\left(\sqrt{2 \mathrm{~g}_{\mathrm{rd}}^{2} \mathrm{~d}_{\mathrm{rd}}^{-\mathrm{m}} \mathrm{E}_{\mathrm{r}} / \mathrm{N}_{0}}+\frac{2 \mu_{01}}{\sqrt{2 \mathrm{~g}_{\mathrm{rd}}^{2} \mathrm{~d}_{\mathrm{rd}}^{-\mathrm{m}_{\mathrm{r}}} \mathrm{E}_{\mathrm{r}} / \mathrm{N}_{0}}}\right)$

- Derivation of $\Psi_{2}\left(\mu_{0 j}\right)$ : Substituting $\mathrm{i}=0$ and $\mathrm{j}=2$ into (28) yields

$\left\|\mathrm{G}\left(\mathrm{T}_{0}-\mathrm{T}_{2}\right)\right\|=$

$$
\begin{gathered}
\left\|\left[\begin{array}{lll}
g_{s d 1} \sqrt{d_{s d}^{-m}} & g_{s d 2} \sqrt{d_{s d}^{-m}} & 0 \\
g_{s d 2}^{*} \sqrt{d_{s d}^{-m}} & -g_{s d}^{*} \sqrt{d_{s d}^{-m}} & 0 \\
0 & 0 & g_{r d} \sqrt{d_{r d}^{-m}}
\end{array}\right]\left[\begin{array}{lll}
0 & 2 \sqrt{E_{s}} & 0
\end{array}\right]^{T}\right\| \\
=\sqrt{4 g_{s d 2}^{2} d_{s d}^{-m} E_{s}+4 g_{s d 1}^{2} d_{s d}^{-m} E_{s}}(33)
\end{gathered}
$$

Accordingly, $\Psi_{2}\left(\mu_{0 \mathrm{j}}\right)$ is given by

$$
\begin{array}{r}
\Psi_{2}\left(\mu_{0 j}\right)=Q\left(\sqrt{\frac{2 g_{s d 1}^{2} d_{s d}^{-m} E_{s}}{N_{0}}+\frac{2 g_{s d 2}^{2} d_{s d}^{-m} E_{s}}{N_{0}}}\right. \\
\left.+\frac{2 \mu_{02}}{\sqrt{\frac{2 g_{s d 1}^{2} d_{s d}^{-m} E_{s}}{N_{0}}+\frac{2 g_{s d 2}^{2} d_{s d}^{-m} E_{s}}{N_{0}}}}\right)
\end{array}
$$

- Derivation of $\Psi_{3}\left(\mu_{0 j}\right) \quad$ : Substituting $\mathrm{i}=0$ and $\mathrm{j}=3$ into (28) yields

$\left\|\mathrm{G}\left(\mathrm{T}_{0}-\mathrm{T}_{3}\right)\right\|=$

$$
\begin{aligned}
& \left\|\left[\begin{array}{ccc}
g_{s d 1} \sqrt{d_{s d}^{-m}} & g_{s d} \sqrt{d_{s d}^{-m}} & 0 \\
g_{s d 2}^{*} \sqrt{d_{s d}^{-m}} & -g_{s d}^{*} \sqrt{d_{s d}^{-m}} & 0 \\
0 & 0 & g_{r d} \sqrt{d_{r d}^{-m}}
\end{array}\right]\left[\begin{array}{lll}
0 & 2 \sqrt{E_{s}} & 2 \sqrt{E_{r}}
\end{array}\right]^{T}\right\| \\
& =\sqrt{2 g_{r d}^{2} d_{r d}^{-m} E_{r}+4 g_{s d 2}^{2} d_{s d}^{-m} E_{s}+4 g_{s d 1}^{2} d_{s d}^{-m} E_{s}}(35)
\end{aligned}
$$

Accordingly, $\Psi_{3}\left(\mu_{0 \mathrm{j}}\right)$ is given by

$$
\begin{aligned}
& \Psi_{3}\left(\mu_{0 j}\right)= \\
& Q\left(\sqrt{\frac{2 g_{r d}^{2} d_{r d}^{-m} E_{r}+4 g_{s d 2}^{2} d_{s d}^{-m} E_{s}+4 g_{s d 1}^{2} d_{s d}^{-m} E_{s}}{N_{0}}}+\frac{2 \mu_{03}}{\sqrt{\frac{2 g_{r d}^{2} d_{r d}^{-m} E_{r}+4 g_{s d 2}^{2} d_{s d}^{-m} E_{s}+4 g_{s d 1}^{2} d_{s d}^{-m} E_{s}}{N_{0}}}}\right)(
\end{aligned}
$$

- Derivation of $\Psi_{4}\left(\mu_{0 j}\right) \quad$ : Substituting $\mathrm{i}=0$ and $\mathrm{j}=6$ into (28) yields

$$
\begin{gathered}
\left.\left\|G\left(T_{0}-T_{6}\right)\right\|=\|\| \begin{array}{ccc}
g_{s d 1} \sqrt{d_{s d}^{-m}} & g_{s d 2} \sqrt{d_{s d}^{-m}} & 0 \\
g_{s d 2}^{*} \sqrt{d_{s d}^{-m}} & -g_{s d 1}^{*} \sqrt{d_{s d}^{-m}} & 0 \\
0 & 0 & g_{r d} \sqrt{d_{r d}^{-m}}
\end{array}\right]\left[\begin{array}{lll}
2 \sqrt{E_{s}} & 2 \sqrt{E_{s}} & 0
\end{array}\right]^{\top} \| \\
=\sqrt{8 g_{s d 2}^{2} d_{s d}^{-m} E_{s}+8 g_{s d 1}^{2} d_{s d}^{-m} E_{s}}
\end{gathered}
$$

Accordingly, $\Psi_{4}\left(\mu_{0 \mathrm{j}}\right)$ is given by

$$
\Psi_{4}\left(\mu_{0 \mathrm{j}}\right)=
$$

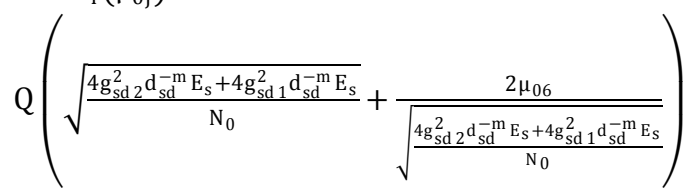

- Derivation of $\Psi_{5}\left(\mu_{0 \mathrm{j}}\right) \quad$ : Substituting $\mathrm{i}=0$ and $\mathrm{j}=7$ into (28) yields

$\left\|\mathrm{G}\left(\mathrm{T}_{0}-\mathrm{T}_{7}\right)\right\|$

$=\left\|\left[\begin{array}{ccc}g_{\text {sd } 1} \sqrt{d_{s d}^{-m}} & g_{s d} \sqrt{d_{s d}^{-m}} & 0 \\ g_{s d 2}^{*} \sqrt{d_{s d}^{-m}} & -g_{s d}^{*} \sqrt{d_{s d}^{-m}} & 0 \\ 0 & 0 & g_{\text {rd }} \sqrt{d_{r d}^{-m}}\end{array}\right]\left[\begin{array}{lll}2 \sqrt{E_{s}} & 2 \sqrt{E_{s}} & 2 \sqrt{E_{r}}\end{array}\right]^{T}\right\|$

$$
=\sqrt{8 g_{s d 2}^{2} d_{s d}^{-m} E_{s}+8 g_{s d 1}^{2} d_{s d}^{-m} E_{s}+4 g_{r d}^{2} d_{r d}^{-m} E_{r}}
$$

Let us define the parameter $\epsilon_{07}$ as follows

$\epsilon_{07}=\sqrt{8 g_{\text {sd } 2}^{2} d_{s d}^{-m} E_{s}+8 g_{s d}^{2} d_{s d}^{-m} E_{s}+4 g_{r d}^{2} d_{r d}^{-m} E_{r}}$

Accordingly, $\Psi_{5}\left(\mu_{0 \mathrm{j}}\right)$ is given by

$\Psi_{5}\left(\mu_{0 \mathrm{j}}\right)=Q\left(\frac{\epsilon_{07}}{\sqrt{2 \mathrm{~N}_{0}}}+\frac{2 \mu_{07} \sqrt{2 \mathrm{~N}_{0}}}{\epsilon_{07}}\right)$

From previous equations, it is desired to average $\Psi_{\mathrm{k}}(\mathrm{i}, \mathrm{j})\{\forall \mathrm{k}=1,2,3,4,5\}$ over the distribution of the fading gains. Averaging $\Psi_{\mathrm{k}}(\mathrm{i}, \mathrm{j})$ is done using the mathematical technique found in [14], we get

$$
\begin{aligned}
& p\left(w_{k} \rightarrow w_{j}\right)= \\
& \left\{\begin{array}{l}
\frac{1}{2} \sum_{m \in \rho_{k, j}}\left(1-\eta_{m}\right) e^{-2 \mu_{k j}\left(1+\frac{1}{\eta_{m}}\right)} \prod_{\substack{i \in \rho_{k, j} \\
i \neq m}} \frac{\gamma_{m}}{\gamma_{m}-\gamma_{i}} \text { if } \mu_{k j} \geq 0 \\
\sum_{m \in \rho_{k, j}}\left(1-\frac{1+\eta_{m}}{2}\right) e^{-2 \mu_{k j}\left(1-\frac{1}{\eta_{m}}\right)} \prod_{\substack{i \in \rho_{k, j} \\
i \neq m}} \frac{\gamma_{m}}{\gamma_{m}-\gamma_{i}} \text { if } \mu_{k j}<0
\end{array}\right.
\end{aligned}
$$




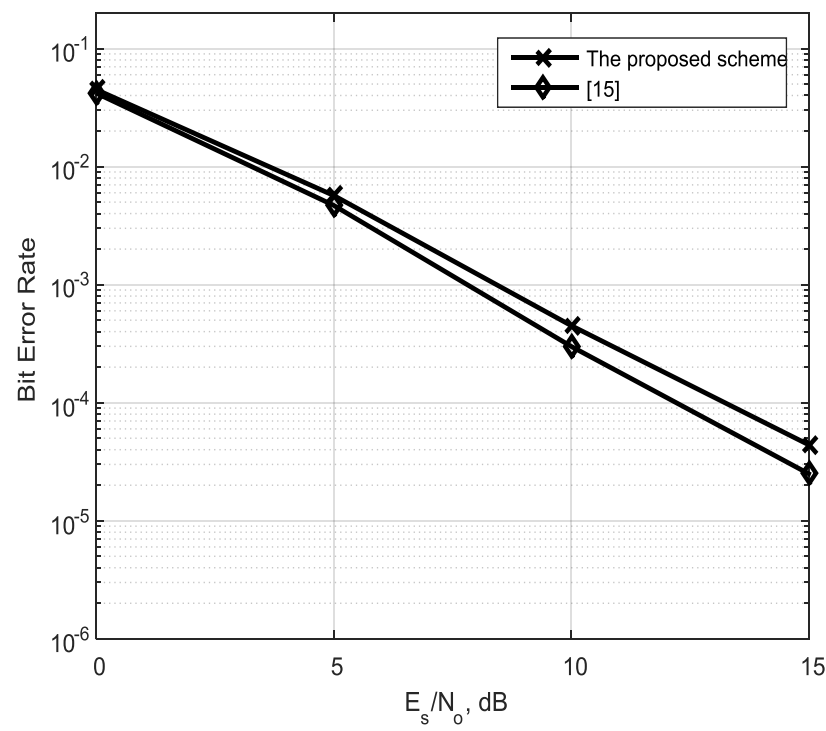

Fig. 2 BER of the proposed scheme and [15]

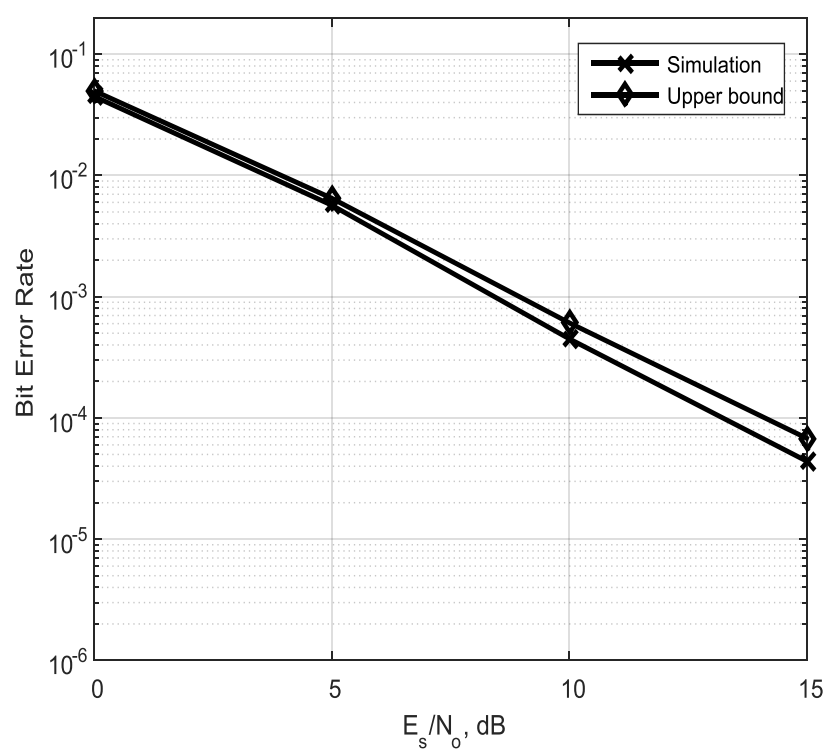

Fig. 3 BER comparison between the upper bound and simulation result

The set $\rho_{\mathrm{k}, \mathrm{j}}$ consists of all terms that are included in the vector $\left\|G\left(T_{k}-T_{j}\right)\right\|$. For example the vector $\left\|G\left(T_{0}-T_{7}\right)\right\|$, according to (39), has three terms, $\gamma_{1}=2 \frac{d_{s d}^{-m} E_{s}}{N_{0}}, \gamma_{2}=$ $2 \frac{d_{s d}^{-m} E_{s}}{N_{0}}, \gamma_{3}=\frac{d_{r d}^{-m} E_{r}}{N_{0}}$, and $\eta_{m}=\sqrt{\frac{\gamma_{m}}{1+\gamma_{m}}}$

\section{Simulation results and discussions}

In this section, both the analysis results that are given by (42) and the simulations results that are given by (21) are introduced. Figure 2 shows the comparison of the bit error performance between the proposed scheme and that is founded in [15]. In the simulation result shown in Figure 2, it is assumed that the source transmitted $S N R E_{S} / N_{o}$ is equal to the relay transmitted $S N R E_{r} / N_{o}$ and all links distances are assumed to be $1 \mathrm{~m}$ without effect on the generality of the proposed scheme in this simulation. The bit
Table 2: Summary of the comparison between the proposed scheme

\begin{tabular}{|l|l|l|}
\multicolumn{3}{|c|}{ and [15] } \\
\hline Scheme & \multicolumn{1}{|c|}{$[\mathbf{1 5}]$} & The proposed scheme \\
\hline Codebook size & 16 Codewords & 8 Codewords \\
\hline Spectrum efficiency & 4 used channels & 3 used channels \\
\hline Bit error rate & $\begin{array}{l}{[15] \text { is less than the proposed scheme by }} \\
\text { about .5dB }\end{array}$ \\
\hline
\end{tabular}

error rate is plotted against the source transmitted SNR. The results indicate that the performance of the proposed scheme and [15] is much closer together especially in low values of SNR. From Figure 2, it is clear that [15] has a bit error rate less than that is found in the proposed scheme because it uses a number of channels larger than that are used in the proposed scheme, although the proposed scheme has better spectrum efficiency as the relay uses only one channel while it uses two channels in [15] to send its data to the receiver. Another feature of the proposed scheme is founded in its complexity when it is compared with [15]. Because in [15], the MAP decoder should estimate the transmitted codeword from 16 codewords because the receiver receives four signals, two signals from the source and two signals from the relay, while in the proposed scheme the MAP decoder should estimate the transmitted codeword from only 8 codewords as shown in (21). Therefore, the proposed scheme has less complexity than [15]. Figure 3 shows the bit error rate obtained from the simulation given by (21) and that is obtained from the upper bound given by (42) under the same simulation parameters used in Figure 2. The upper bound (42) can be used in performance analysis of the considered network and poweroptimization for the cooperative network. Derivation of the upper bound is a straightforward process and it can be applied to any cooperative network having any number of sources and relays to improve the overall performance for the network under consideration.

The relay positioning issue is demonstrated in Figure 4. Figure 4 shows the system BER against the distance between the relay and the receiver $\mathrm{d}_{\mathrm{sr}}$. Assuming that the distance between the source and the receiver $d_{s d}$ is equal 2 and the sum of distances between the source and the relay $d_{s r}$ and the distance between the relay and the receiver $d_{r d}$ is equal 2. Three different cases are considered here. In the first case, the source transmitted $S N R E_{S} / N_{o}$ is equal to the relay transmitted $\mathrm{SNRE}_{\mathrm{r}} / \mathrm{N}_{\mathrm{o}}$. In the second case, the source transmitted SNR $E_{S} / N_{o}$ is greater than the relay transmitted SNR $E_{r} / N_{o}$. In the third case, the source transmitted SNR $E_{S} / N_{o}$ is less than the relay transmitted $S N R E_{r} / N_{o}$. It is clear from Figure 4 that more transmitted power is needed to be allocated to the relay when it is far from the receiver. Moreover, when the transmitted SNR is equal for the source and the relay, the relay isn't allocated in the midway between the source and the receiver as expected because the source has two transmitting antennas. The summary of the comparison between the proposed scheme and [15] is introduced in Table 2. This summary shows that the proposed scheme offers a further level of superiority over the previous related work.

\section{Conclusions}

In this work, we presented a new approach for the cooperative network using the MAP decoder and the Alamouti code decoding schemes for the MISO wireless network that uses the DF protocol as a cooperation protocol. 


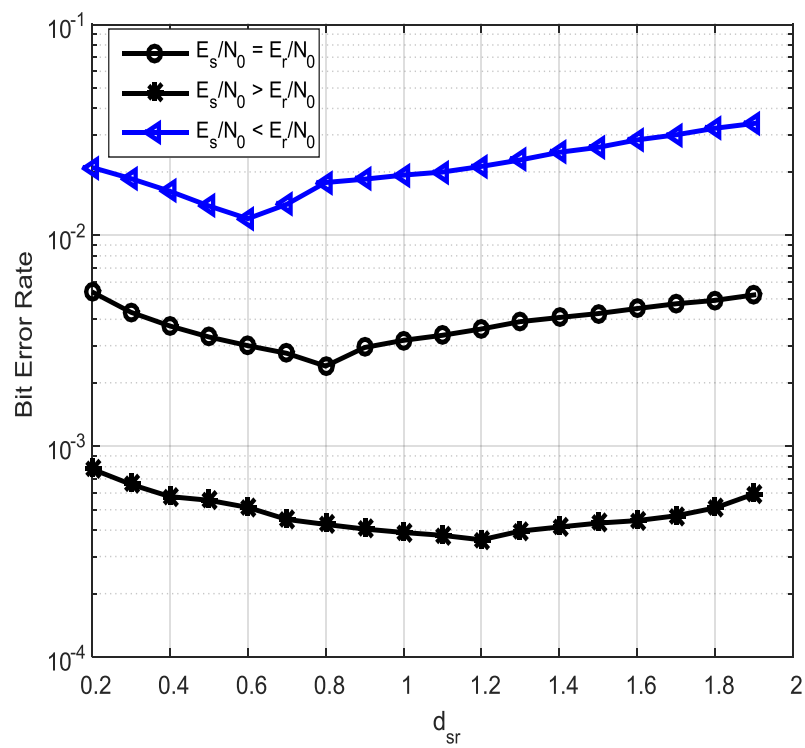

Fig. 4 BER against $d_{\text {sr }}$

A closed form expression for the bit error probability is achieved. The obtained upper bound expression can be used forthe power optimization, relay location selector issue. The obtained results show that the proposed scheme has two advantages over [15] since it has less complexity and better spectrum efficiency because the proposed scheme uses a number of channels less than that are used in [15]. The simulation results that are presented in this work show that the proposed scheme is very efficient and straightforward and can be applied to any cooperative network having any number of sources and relays.

\section{References}

1. J. N. Laneman, D. N. C. Tse, G. W. Wornell, Cooperative diversity in wireless networks: efficient protocols and outage behavior. IEEE Trans. Inf. Theory. 50, 3062-3080 (2004).

2. J. S. Chen, J. X. Wang, Cooperative transmission in wireless networks using incremental opportunistic relaying strategy. IET Commun. 3, 1948-1957 (2009).

3. Q. Li, R. Q. Hu, Y. Qian, G. Wu, Cooperative communications for wireless networks: techniques and applications in LTE-advanced systems. IEEE Wirel. Commun. 19, 22-29 (2012).

4. Y. G. Kim, N. C. Beaulieu, Exact BEP of decode-and-forward cooperative systems with multiple relays in Rayleigh fading channels. IEEE Trans. Veh.Technol. 64, 823-828 (2015).

5. P. Clarke, R. C. de Lamare, Transmit diversity and relay selection algorithms for multirelay cooperative MIMO systems. IEEE Trans. Veh. Technol. 61, 1084-1098 (2012)

6. Andrea Goldsmith, "Wireless communications", Cambridge University Press, 2005

7. T. A. Khalaf, "Security and Prioritization in Multiple Access Relay Networks”, Ph.D.dissertation, Dept. Elec., Univ. of Iowa State, USA,2011.

8. M. Iezzi, M. Di Renzo, and F. Graziosi "On Diversity Order and Coding Gain of

Multisource Multirelay Cooperative Wireless Networks With Binary Network Coding”, ,IEEE Trans.Veh. Technol., Vol. 62, No. 3,March 2013.

9. A. Nasri,R. Schober, M. Uysal, "Performance and Optimization of Network-Coded Cooperative Diversity Systems," IEEE Trans. Commu., Vol.61, No.99, pp.1111 - 1122, 2013

10. M. D. Selvaraj, R. K. Mallik, Error analysis of the decode and forward protocol with selection combining. IEEE Trans. Wirel. Commun. 8, 3086-3094 (2009).

11. H. Mohammed, T. A. Khalaf, in 2013 9th International Computer Engineering Conference (ICENCO). Optimal positioning of relay device in wireless cooperative communication networks, (2013), pp. 122-127. https://doi. org/10.1109/icenco.2013.6736487

12. N. Tavakkoli, P. Azmi, N. Mokari, Optimal positioning of relay device in cooperative molecular communication networks. IEEE Trans. Commun. 65, 5293-5304 (2017)

13. Qian Li; R.Q. $\mathrm{Hu}, \mathrm{Yi}$ Qian, and Geng $\mathrm{Wu}$, "Cooperative communications for wireless networks: techniques and applications in LTE-advanced systems," IEEE Wireless Communications, Vol. 19, No. 2, pp. 22-29, April 2012

14. T. A. Khalaf, S. M. Ramzy, Decoding scheme for relay networks with parity forwarding cooperation protocol. IET Commun. 8, 13171324 (2014).

15. Taha A. Khalaf1, HazemMohammed"Bit error rate performance analysis of AC-MAP in multiple input single output wireless relay network" URASIP Journal on WirelessCommunications and Networking (2020) 2020:12

16. Z. Mingjie, L. Bihong, in 2006 7th International Symposium on Antennas,Propagation EM Theory. Performance analysis of Alamouti scheme with imperfect multiple transmit antennas selection in Rayleigh fading channel, (2006), pp. 1-4. https://doi.org/10.1109/isape.2006.353529 